\title{
Analytical investigation of a novel interrogation approach of fiber Bragg grating sensors using Optical Frequency Domain Reflectometry
}

\author{
Kivilcim Yüksel*, Deniz Pala \\ Electronics Engineering and Telecommunication Department, Izmir Institute of Technology, IZTECH, Gülbahce Kampüsü, 35430 Izmir, Turkey
}

\section{A R T I C L E I N F O}

\section{Article history:}

Received 9 September 2015

Received in revised form

27 December 2015

Accepted 5 January 2016

Available online 13 February 2016

Keywords:

Optical Frequency Domain Reflectometry

Fiber Bragg grating

Transverse strain

Composite material

Fiber optic sensors

\begin{abstract}
A B S T R A C T
This work presents a novel approach in interrogating Polarization Dependent Loss (PDL) of cascaded identical FBGs using Optical Frequency Domain Reflectometer (OFDR). The fundamentals of both polarisation properties of uniform FBGs and polarisation-sensitive OFDR are explained and the benefits of this novel approach in measuring transversal load are discussed. The numerical programs computing the spectral evolution of PDL of the FBGs in the array as a function of grating parameters (grating length and birefringence) are presented. Our simulation results show an excellent agreement with the previously reported simulation (and experimental) results in the literature obtained on a single FBG by using classical state-of-the-art measurement techniques. As an envisaged application, the proposed system shows the feasibility of measuring the residual stresses during manufacturing process of composite materials which is not straightforward by amplitude spectrum measurements and/or considering only the axial strains.
\end{abstract}

(c) 2016 Elsevier Ltd. All rights reserved.

\section{Introduction}

Optimal efficiency, longer service life, safety, reduced costs together with reduced energy consumption are major concerns for today's science and technology. For many industrial sectors such as transportation, aerospace, and renewable energy (wind turbines), this concerns can be addressed by lightening the structure of mechanical pieces while keeping intact the important requirements of mechanical performance of the final product. It is made possible by replacing critical parts and high stress members (airplane wings, unmanned air vehicles, wind turbines) by composite materials. However, the unique mechanical properties of composite materials (i.e. superior strength, durability, and corrosion resistance) cannot be fully exploited without monitoring them when material is placed under constraints. This is because the behavior of composite materials under loading is different from metals and can rapidly degrade when internal damage occurs. It is therefore essential to monitor their behavior during manufacturing, prototyping and in the field for the purpose of improved performance, improved safety, and reduced cost.

Residual stresses appearing during fabrication process (curing) is of particular interest for composite materials as they may weaken the structure and increase the risk of sudden breakage. Fiberreinforced composites are indeed fragile in out-plane-direction

\footnotetext{
* Corresponding author. Tel.: +90232750 6540 .

E-mail address: kivilcimyuksel@iyte.edu.tr (K. Yüksel).
}

because of their anisotropic nature and can be damaged by excessive transverse loads. Many groups have been working to develop novel technologies based on Fiber Optic Sensors (FOS) for monitoring production steps and strain distribution of the composite structures. Among these studies, Fiber Bragg grating (FBG) sensors have brought about a revolutionary dimension to the research in this field. FBGs are low-cost, mass producible intrinsic sensing devices providing self-referencing and wavelengthencoded linear response to the physical parameter to be measured. Being photo-imprinted in the core of an optical fiber, FBGs not only benefit from all the advantages of FOS but they also offer an important instrumentation capability which is not possible with conventional sensors: quasi-distributed and embedded sensing. Quasi-distributed sensing involves several concatenated FBGs on a single fiber that can be analyzed with a single interrogating system. In this context, a fast, reliable and cost-effective interrogation unit that can be implemented in many application areas is of paramount importance for FBG-based sensing systems.

In terms of interrogation tools presented in the literature, two main tendencies can be recognized. The first group consists of solutions where the sensing points (FBGs) are illuminated by a broadband light source. The light reflected by the FBGs with different wavelengths is then analyzed by a wavelength detection system (either by the way of wavelength-selective passive components or by employing actives techniques such as wavelength tracking, scanning, and interferometry) [1-4]. In these most commercially encountered systems, a unique range of operating wavelength is dedicated to each grating of the concatenation. 
Therefore, the number of sensing points is directly limited by the spectral ranges of the source and the detector, and the wavelength spacing between two gratings. The second group of solutions is based on the use of optical reflectometry techniques. Optical Time Domain Reflectometry (OTDR) and coherent Optical Frequency Domain Reflectometry (OFDR) techniques are the two main candidates that can be exploited in the framework of optical sensing. In terms of equipment availability and cost, OTDR is a standard, off-the-shelf tool with accessible prices but brings about two big limitations related to the inevitable dead-zone and the long measurement time. OFDR on the other hand tackles the disadvantages of OTDR and has been nowadays gaining a renewed interest as an interrogating tool for use in the quasi-distributed sensing field [5].

Thanks to the use of a linearly swept optical source together with the proper demodulation algorithms, Optical FrequencyDomain Reflectometry (OFDR) reportedly allows recovering the reflected spectrum of the FBGs placed in a sensor array even if the identical FBGs are used. The amplitude spectrum of the identical FBGs is interrogated by OFDR to address the effect of an axial strain [6] or temperature changes [7]. The amplitude spectrum however cannot provide the sufficient information in the case of transversal load measurements. Unlike the axial strain and temperature effects which produce linear shift in the center wavelength of a FBG, transverse strain induces birefringence effect that results in two distinct Bragg wavelengths. This split might be as low as a few picometers making it difficult to be distinguished by optical spectrum analyzer (OSA) or other FBG sensor interrogation systems. It has been recently shown that tracking (in wavelength and amplitude) the Polarization Dependent Loss (PDL) of FBGs can provide more significant information for transversal load measurements. Still, PDL measurements in this context has been addressed in a very limited number of publications which proposes the use of specific (High birefringence [8] or microstructured [9]) optical fibre and very expensive commercial interrogation tools. Caucheteur et al. recently reported the temperature-insensitive transverse strain measurements by means of tracking the maximum PDL values of a uniform FBG written into a standard single-mode fiber [10]. However none of these transverse strain sensors using FBG could investigate quasidistributed and/or distributed measurement capability which significantly limits their implementations into composite materials [11].

In order to overcome this problem, Murayama et al. introduced a novel concept in realizing distributed measurements by using long-length FBG interrogated by OFDR and successfully implemented this technique to several applications (e.g. damage detection in adhesive bondings, distributed strain measurements [12], and birefringent FBG response measurements [13]). The same research team also realized lateral load measurements by discriminating the Bragg peaks corresponding to both polarization modes $[14,15]$. Observing the so-called beat cycle, very low load values $2 \mathrm{~N} / \mathrm{mm}$ compared to previous literature was reportedly measured which would be very useful in addressing residual strains in composite material fabrication stages.

In this paper, we build a numerical simulation model for studying the optical response of the FBG array interrogated by the OFDR. The numerical programs allow computing the spectral evolution of Polarization Dependent Loss (PDL) of the FBGs in the array as a function of system parameters (grating parameters, wavelength range, the distance between the grating sensors, etc.). Our simulation results show an excellent agreement with the previously reported simulation (and experimental) results in the literature obtained on a single FBG by using classical state-of-theart measurement techniques $[10,16]$. The original contribution of this paper is the exploitation the polar properties of the identical uniform FBGs in a quasi-distributed manner. This will permit us to measure the transversal strain which is not possible by amplitude measurements. The method proposed in this paper can also be readily used with long-length FBG in a distributed manner (complementary to the work of Wada et al.). This theoretical study will serve to design appropriate gratings for the development of novel sensor applications. The particular envisaged application would be monitoring of resin injection and curing stages of composite structure manufacturing (e.g. measure the residual stresses and dry spots).

\section{Numerical simulation model of polarisation sensitive OFDR}

\subsection{Fiber Bragg gratings}

In its simplest form, a FBG is a permanent and periodic modification of the core refractive index value along an optical fiber. An FBG is described by the following parameters [17]:

- The length $L$ over which the variation on the refractive index is realised. The typical grating length ranges from a few $\mathrm{mm}$ up to a few tens of $\mathrm{cm}$.

- The periodicity $\Lambda$ (also called grating pitch) ranges from $200 \mathrm{~nm}$ to $800 \mathrm{~nm}$.

- The amplitude of the refractive index modulation $\delta n$ typically ranges from $10^{-5}$ to $10^{-3}$.

The inscription of such a perturbation in the fiber core refractive index induces mode coupling between two counter propagating beams. That coupling occurs for the resonance wavelength of the grating, the Bragg wavelength, obtained by

$\lambda_{\text {Bragg }}=2 n_{\text {eff }} \Lambda$

where $n_{\text {eff }}$ is the effective refractive index of the core at the Bragg wavelength $\lambda_{\text {Bragg. }}$.

Exposing one side of the fiber to the UV light source, during the fabrication process of fiber Bragg grating, induces variation of refractive index along the cross section of fiber. The refractive index becomes larger at the core side which is subjected to UV laser. Refractive index variation produces photo-induced birefringence (order of magnitude varies between $10^{-6}$ and $10^{-5}$ in practice). Combination of photo-induced birefringence with the intrinsic fiber birefringence results a global birefringence value $\Delta \beta$.

The differences between effective refractive indices of the two polarization modes are expressed as,

$n_{\text {eff }, \mathrm{x}}=n_{\text {eff }}+\frac{\Delta \beta}{2}$

$n_{\mathrm{eff}, \mathrm{y}}=n_{\mathrm{eff}}-\frac{\Delta \beta}{2}$

where, as stated before, $\Delta \beta$ is the global birefringence value reached at the end of the writing process.

\subsection{FBG array interrogated by OFDR}

In the literature, to simulate the interrogation of a concatenation of identical FBGs with OFDR, a variation of the couple mode theory [18] or the transfer matrices to model a FBG array and OFDR system [19] have been employed. In the framework of this paper, we elaborated the piece-wise uniform approach in an original way using both the coupled mode theory and Jones formalism considering two polarisation modes. For this purpose, OFDR traces are simulated and transmission coefficients of the FBGs for both 


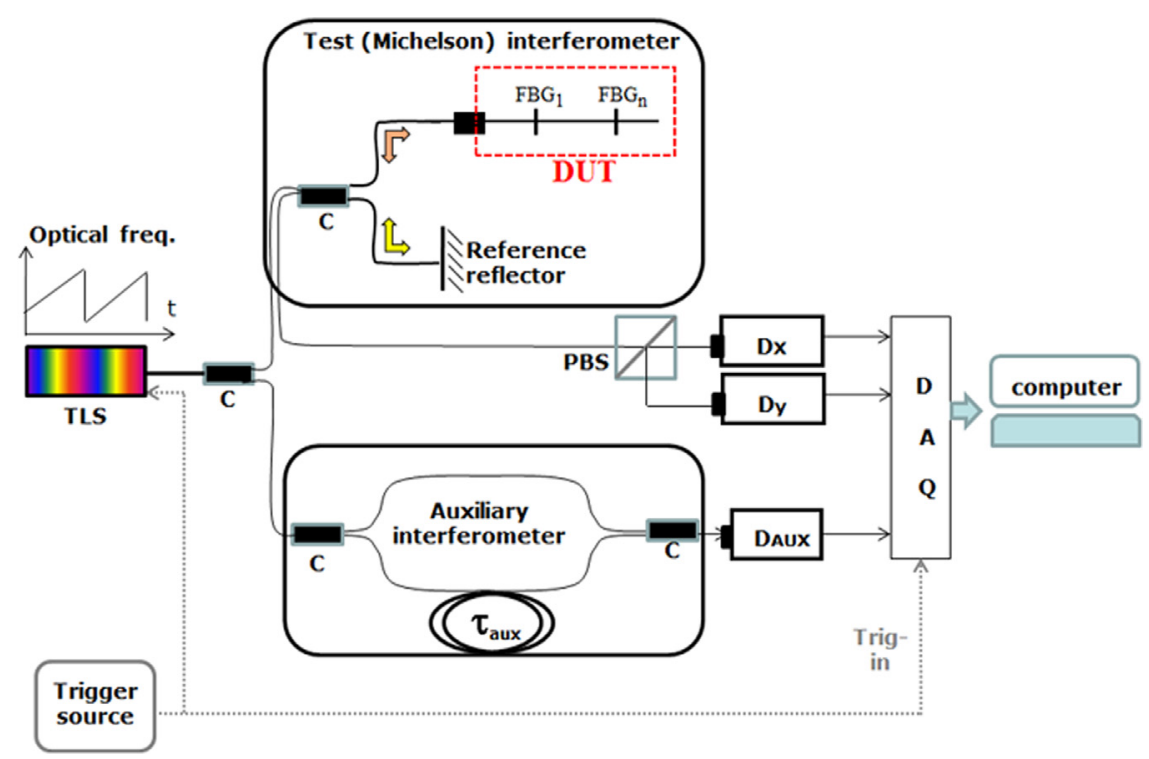

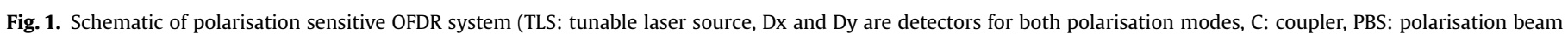
splitter, DAQ: data acquisition card).

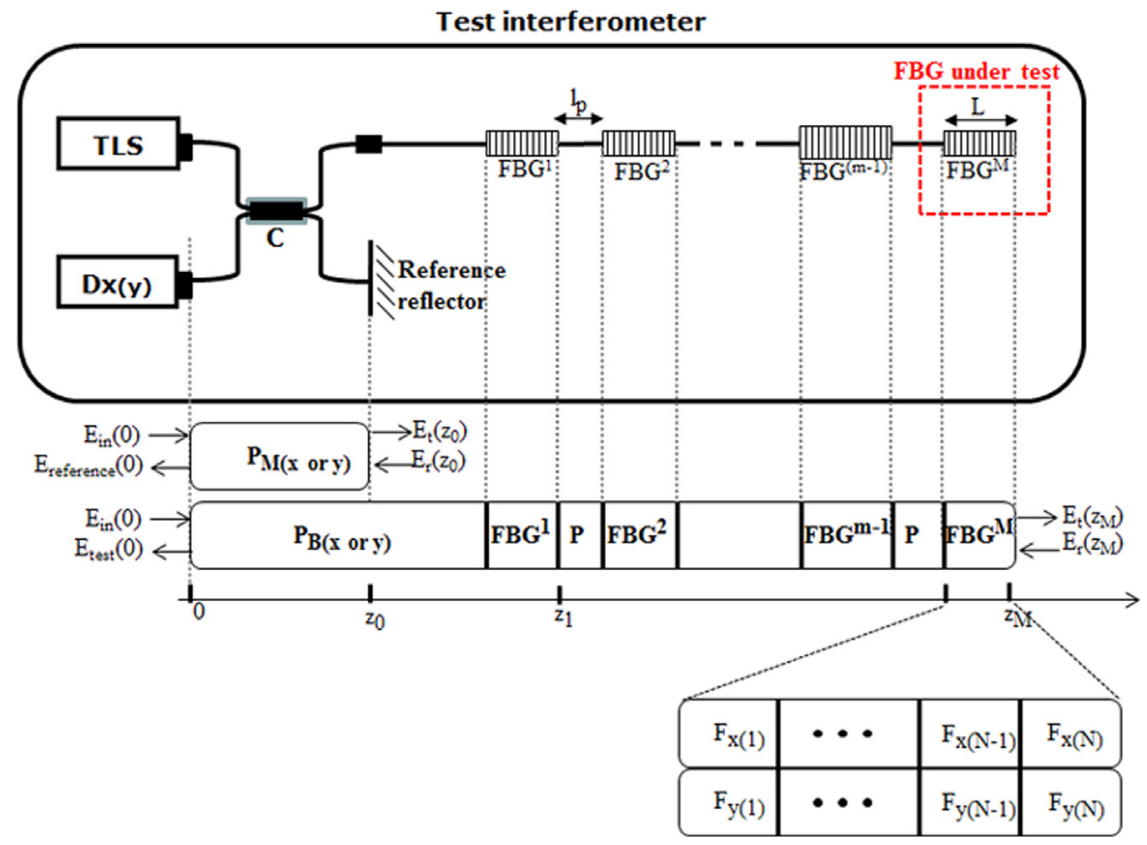

Fig. 2. Schematic of the test interferometer for the numerical simulation model.

polarisation modes are demodulated through several polarization states. Finally, based on the demodulated transmission coefficients $\left(T_{x}\right.$ and $\left.T_{y}\right)$, PDL of the FBGs are obtained using

$P D L(\lambda)=\left|10 \log _{10} \frac{T_{x}(\lambda)}{T_{y}(\lambda)}\right|$

where $T_{x}\left(T_{y}\right)$ is the power transmission coefficient for $x(y)$ polarisation mode. Fig. 1 shows the block diagram of the OFDR interrogating cascaded FBGs. In this configuration, a tunable laser source (TLS) sweeps the wavelength of the continuous-wave signal linearly in time without mode hops. Then, the probe signal is split into two paths, namely the test interferometer and the auxiliary interferometer. The latter is used to compensate the effect of nonlinear frequency sweep to get a spatial resolution in the millimeter range as demonstrated in our previous works [20]. The signal observed on the $D_{\mathrm{AUX}}$ as a function of time is expressed as

$D_{\mathrm{AUX}}=\cos \left(2 \pi \gamma \tau_{\text {aux }} t\right)$

where $\gamma$ is the tuning rate (in $\mathrm{Hz} / \mathrm{s}$ ) and $\tau_{\text {aux }}$ is the optical path difference (in second) of the auxiliary interferometer. Eq. (5) can be re-expressed in the following form:

$D_{\mathrm{AUX}}=\cos \left(2 n_{\mathrm{eff}} L_{\mathrm{aux}} k\right)$

where $L_{\text {aux }}$ optical path length $\left(\tau_{a u x}=c L_{\text {aux }} / n_{\text {eff }}\right), c$ being speed of light in vacuum. $D_{\text {AUX }}$ is used to sample the signal at the both detectors $\left(D_{x}\right.$ and $\left.D_{y}\right)$ at equidistant $k$ intervals, thus compensate the effect of nonlinear sweep [20].

The test interferometer includes the cascaded FBGs in the test arm whereas a reference reflector (also called local oscillator) is placed in the reference arm. The test signal which is reflected back from the FBGs coherently interfere at the coupler with the reference signal returning from the reference reflector. Distortion 
occurs when a concatenation of gratings sharing the same spectral characteristics are addressed simultaneously due to light having to pass through all the FBGs located between the FBG under test and the interrogator (spectral-shadowing crosstalk). This problem, which is analyzed in our previous article [21], has not been taken into account in the present work.

\subsection{Simulation model: cascaded FBGs interrogated by OFDR}

Simulation model is shown in Fig. 2 where transfer matrices are assigned to different sections of the system. For each section, two transfer matrices are used for the two polarisation modes along $x$ and $y$ axes. The transfer matrix of the each FBG in the array is divided into $N$ uniform sections of equal length $(\Delta z)$. The propagation through each of the uniform sections is described by a matrix [17]:

$F_{j}=\left[\begin{array}{cc}\cosh \left(\alpha_{j} \Delta z\right)-i \frac{\delta_{j} \sinh \left(\alpha_{j} \Delta z\right)}{\alpha_{j}} & -i \frac{\kappa \sinh \left(\alpha_{j} \Delta z\right)}{\alpha_{j}} \\ i \frac{\kappa \sinh \left(\alpha_{j} \Delta z\right)}{\alpha_{j}} & \cosh \left(\alpha_{j} \Delta z\right)+i \frac{\delta_{j} \sinh \left(\alpha_{j} \Delta z\right)}{\alpha_{j}}\end{array}\right]$

where $\delta_{j}$ is called self-coupling coefficient and $\kappa$ is the AC coupling coefficient between two modes propagating in opposite directions, and $\nu$ represents the visibility of the interference fringes $(0<\nu<1)$. These parameters are defined by the following relationships

$\alpha_{j}=\sqrt{\kappa^{2}-\delta_{j}^{2}}$

$\kappa=\frac{\pi}{\lambda} \nu \delta n$

$\delta_{j}=\frac{2 \pi n_{\mathrm{eff}, \mathrm{j}}}{\lambda}-\frac{\pi}{\Lambda}$

The phase-shift matrix of the reference $\operatorname{arm}\left(P_{M j}, j=x\right.$ or $\left.y\right)$ is given as

$P_{M j}=\left[\begin{array}{cc}\exp \left[-i \phi_{j} / 2\right] & 0 \\ 0 & \exp \left[i \phi_{j} / 2\right]\end{array}\right]$

$\phi_{j}=\frac{4 \pi}{\lambda} n_{\mathrm{eff}, \mathrm{j}} z_{0}$

For the reference arm containing the mirror, relation between output and input of the system is given as (the dependency on $x$ and $y$ modes are not indicated for the sake of simplicity),

$\left[\begin{array}{c}E_{\text {in }}(0) \\ E_{\text {reference }}(0)\end{array}\right]=P_{M}\left[\begin{array}{c}E_{\mathrm{t}}\left(z_{0}\right) \\ E_{\mathrm{r}}\left(z_{0}\right)\end{array}\right]$

where $E_{\text {in }}(0)$ and $E_{\mathrm{t}}\left(z_{0}\right)$ are the amplitudes of the forward propagation mode at the input and at the mirror position; whereas $E_{\text {reference }}(0)$ and $E_{\mathrm{r}}\left(z_{0}\right)$ are the amplitudes of the backward propagating mode at the input and at the mirror position respectively (refer to Fig. 2). When the boundary conditions are applied,

$E_{\text {in }}(0)=1$

$E_{\mathrm{r}}\left(z_{0}\right)=E_{\mathrm{t}}\left(z_{0}\right) e^{i \pi}$

$\left[\begin{array}{c}1 \\ E_{\text {reference }}(0)\end{array}\right]=\left[\begin{array}{ll}P_{\mathrm{Mj} 11} & P_{\mathrm{Mj} 12} \\ P_{\mathrm{Mj} 21} & P_{\mathrm{Mj} 22}\end{array}\right]\left[\begin{array}{c}E_{\mathrm{t}}\left(z_{0}\right) \\ E_{\mathrm{t}}\left(z_{0}\right) e^{i \pi}\end{array}\right]$

the mirror reflection coefficient is calculated using (14)-(16) as

$R L_{\text {Mirror }}=E_{\text {reference( } \mathrm{x})}(0) \mathbf{x}+E_{\text {reference(y) }}(0) \mathbf{y}$

$$
=\frac{P_{\mathrm{Mx} 21}+P_{\mathrm{Mx} 22} e^{i \pi}}{P_{\mathrm{Mx} 11}+P_{\mathrm{Mx} 12} e^{i \pi}} \mathbf{x}+\frac{P_{\mathrm{My} 21}+P_{\mathrm{My} 22} e^{i \pi}}{P_{\mathrm{My} 11}+P_{\mathrm{My} 12} e^{i \pi}} \mathbf{y}
$$

The phase-shift matrix for the fiber section placed in the test arm before the FBG array $\left(P_{\mathrm{Bj}}, j=x\right.$ or $\left.y\right)$ is given as

$P_{\mathrm{Bj}}=\left[\begin{array}{cc}\exp \left[-i \phi_{j} / 2\right] & 0 \\ 0 & \exp \left[i \phi_{j} / 2\right]\end{array}\right]$

$\phi_{j}=\frac{4 \pi}{\lambda} n_{\mathrm{eff}, \mathrm{j}} z_{1}$

For the optical path containing the FBG array, the transfer matrix $T$ can be considered as a multiplication of the transfer matrices corresponding to the fiber section before FBGs $\left(P_{\mathrm{Bj}}\right)$, individual FBGs, and fiber sections between FBGs $(P)$. Assuming equal distances between FBGs, the transfer matrix of the whole system is then expresses as

$T_{\text {Reflection }, \mathrm{j}}=\left[P_{\mathrm{Bj}}\right]\left[F B G_{\mathrm{j}}^{1}\right][P]\left[F B G_{\mathrm{j}}^{2}\right][P] \ldots\left[F B G_{\mathrm{j}}^{m-1}\right][P]\left[F B G_{\mathrm{j}}^{M}\right]$

where

$P_{j}=\left[\begin{array}{cc}\exp \left[-i \frac{4 \pi}{\lambda} n_{\mathrm{eff}, \mathrm{j}} l_{p}\right] & 0 \\ 0 & \exp \left[i \frac{4 \pi}{\lambda} n_{\mathrm{eff}, \mathrm{j}} l_{p}\right]\end{array}\right]$

$l_{p}$ being the separation length between the FBGs. Therefore, the relation between input and output of the whole system containing $M$ FBGs in cascade for the two polarization mode is defined by

$\left[\begin{array}{c}E_{\mathrm{in}(\mathrm{j})}(0) \\ E_{\text {test }(\mathrm{j})}(0)\end{array}\right]=T_{\text {reflection } \mathrm{j}}\left[\begin{array}{c}E_{\mathrm{t}(\mathrm{j})}\left(z_{M}\right) \\ E_{\mathrm{r}(\mathrm{j})}\left(z_{M}\right)\end{array}\right]$

The reflected light from the FBG (\#M in our case) is expressed as

$R L_{\mathrm{FBG}}=E_{\text {test }(\mathrm{x})}(0) \mathbf{x}+E_{\text {test(y) }}(0) \mathbf{y}=a_{x} \exp \left(i \varphi_{x}\right) \frac{T_{\mathrm{x} 21}}{T_{\mathrm{x} 11}} \mathbf{x}+a_{y} \exp \left(i \varphi_{y}\right) \frac{T_{\mathrm{y} 21}}{T_{\mathrm{y} 11}} \mathbf{y}$

where $a_{x}$ and $\varphi_{j}(j=x$ or $y)$ are the amplitude and phase of the polarization state of the input signal and $T_{\mathrm{j} 21}$ and $T_{\mathrm{j} 11}$ denote the elements of transfer matrix, $T_{\text {reflection,j. }}$.

Finally the output signal can be expressed by taking the absolute square of the signals reflected from reference test paths. The detected signals, $D_{x}$ and $D_{y}$, at two outputs of the polarisation beamsplitter can be calculated as,

$D_{\mathrm{x}}=\left|R L_{\mathrm{mirror}, \mathrm{x}}+R L_{\mathrm{FBG}, \mathrm{x}}\right|^{2}$

$D_{\mathrm{y}}=\left|R L_{\text {mirror, } \mathrm{y}}+R L_{\mathrm{FBG}, \mathrm{y}}\right|^{2}$

\subsection{Demodulation of PDL using polarisation sensitive OFDR}

OFDR interrogates a concatenation on $M$ identical lowreflectivity FBGs with identical Bragg wavelength. As schematically represented in Fig. 2, if grating $M$ is under test, then the physical effect (i.e transversal strain) applied on the FBG will effect the PDL spectrum of it. First of all the composite OFDR signal detected in time domain is converted into frequency (distance) domain. Then, the OFDR signal around the FBG under test is bandpass filtered around a specific frequency (location). The required bandwidth of the bandpass filter is inversely related to the wavelength bandwidth of the FBG under test. By applying inverse Fourier transform on the filtered section of the OFDR trace, the reflection spectrum of each FBG in the array can be independently obtained. (This process is applied on both signals at both detectors separately). The two reflection coefficients for both polarisation modes (obtained using the signals $D_{\mathrm{x}}$ and $D_{\mathrm{y}}$ ) provides the transmission coefficients $T_{\mathrm{x}}$ and $T_{\mathrm{y}}$ which are in turn used to calculate (by applying Eq. (4)) the PDL spectrum of each individual FBG. 


\section{Simulation results and discussion}

Once the mathematical model is established, the influence of two parameters have been investigated by the way of numerical simulations: grating length and birefringence. The complete parameter list used for the simulations is given in Table 1. For each parameter (grating length and birefringence), PDL spectra obtained by our simulations are compared with that of analytical calculations reported in the literature [22,23].

Fig. 3 presents the evolution of the PDL with respect to grating length. As shown on the figure, increasing the grating length has the effect of increasing the peak amplitudes at wavelengths coinciding with the edges of the rejection band of the transmitted spectrum. This comes from the fact that increasing the grating length $(L)$ leads to an increasing amplitude variation on the transmitted spectrum whereas the wavelength spacing between the two eigenmodes is slightly influenced. As a result, for long physical lengths, sharp PDL evolutions with important maximum values are obtained. Simulations reveal that PDL maximum amplitude increase from $0.1 \mathrm{~dB}$ to $3 \mathrm{~dB}$ when $L$ is varied from $0.4 \mathrm{~cm}$ to $1.4 \mathrm{~cm}$. Our simulation results (Fig. $3 \mathrm{~b}$ ) are in a very good agreement with the calculations (Fig. 3a) that we realised based on the analytical formulas given in the literature [22,23].

The influence of the birefringence value is investigated in Fig. 4. For $\Delta \beta$ values less than $10^{-4}$, the maximum PDL value monotonically increase. This is because two transmitted spectra corresponding to the $x$ and $y$ modes overlap. For $\Delta \beta$ values higher than $10^{-4}$, the overlapping between the $T_{\mathrm{x}}$ and $T_{\mathrm{y}}$, spectra is progressively removed leading to a saturation in the maximum PDL

Table 1

Parameters for the numerical simulation.

\begin{tabular}{ll}
\hline Sweep range of laser source & $1538-1539 \mathrm{~nm}$ \\
\hline$n_{\text {eff }}$ & 1.45 \\
$z_{0}$ (mirror location) & $1 \mathrm{~m}$ \\
$z_{M}$ (location of the FBG under test, FBG $\# M$ in the array) & $4 \mathrm{~m}$ \\
$M$ (number of FBGs) & 3 \\
$N$ (number of sections on the FBG under demodulation) & 100 \\
FBG length & $1 \mathrm{~cm}$ \\
Periodicity of the refractive index modulation $(\Lambda)$ & $530 \times 10^{-9}$ \\
Average refractive index modulation & $2 \times 10^{-5}$ \\
Number of Points in the FFT (and IFFT) process & $2^{14}$ \\
\hline
\end{tabular}

values. Still the wavelength spacing between the peaks in PDL spectrum increases. Once again, our simulation results (Fig. 4b) are in a very good agreement with the calculations (Fig. 4a) that we realized based on the analytical formulas given in the literature $[22,23]$.

\section{Conclusion and perspectives}

We demonstrated that the Polarization Dependent Loss (PDL) parameter of each FBG in an array can be accurately interrogated using the polarisation sensitive OFDR. The preliminary quantitative steps presented in this paper are of great interest for system design as well as for system performances evaluation toward realizing a quasi-distributed transverse strain sensor. In order to understand whether the polarisation properties (PDL in our case) of uniform FBGs may be interrogated by the way of OFDR, a numerical simulation model has been built. PDL spectra of individual FBGs in an array have been demodulated by applying signal processing steps (windowing $\longrightarrow$ IFFT) on the OFDR traces. Using the demodulated reflection and transmission spectra of FBGs for both polarisation modes, PDL of a single FBG in the array is obtained independently from others. The influence of the design parameters such as length and birefringence of the FBG are studied. Simulation results compared with the previous work (interrogating the PDL on a single FBG by using conventional devices), shows a very good agreement. The original contribution of this paper is the exploitation the Polarisation Dependent Loss, (PDL) of the identical uniform FBGs in a quasi-distributed manner. Simulation results show that new design in OFDR will make it possible to address these identical FBGs (cheap and easy to handle). The proposed system may be readily applied into a distributed sensor by using long-length FBGs. As an envisaged application, the proposed system shows the feasibility of measuring the residual stresses during manufacturing process of composite materials which is not straightforward by amplitude spectrum measurements and/or considering only the axial strains [24]. Concerning the monitoring of residual deformation during curing process of composite materials by the way of FBGs, there is still a lack of intellectual property that can be transferred to industrial applications. Among the approaches proposed in the literature, there is no universal solution that is standardized for the practical implementations. Recognizing the tremendous increase in the use of
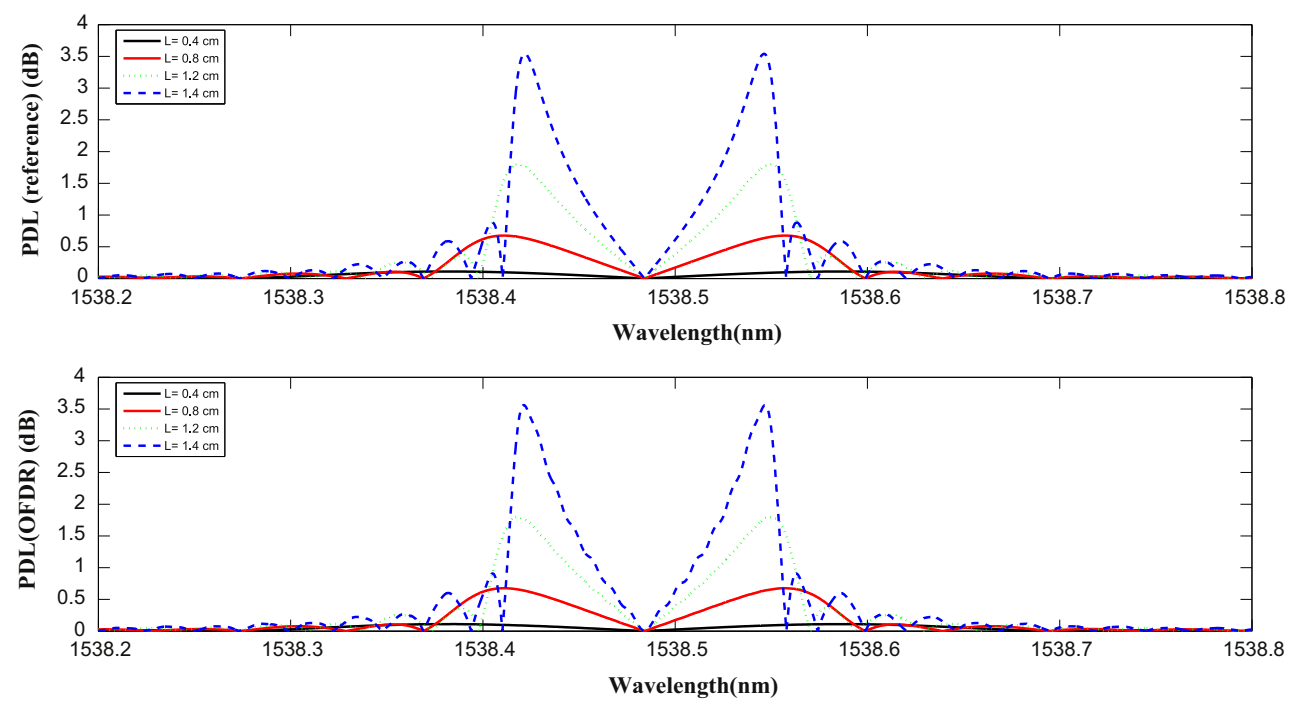

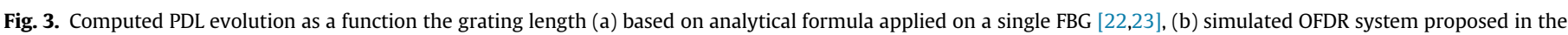
current work. 

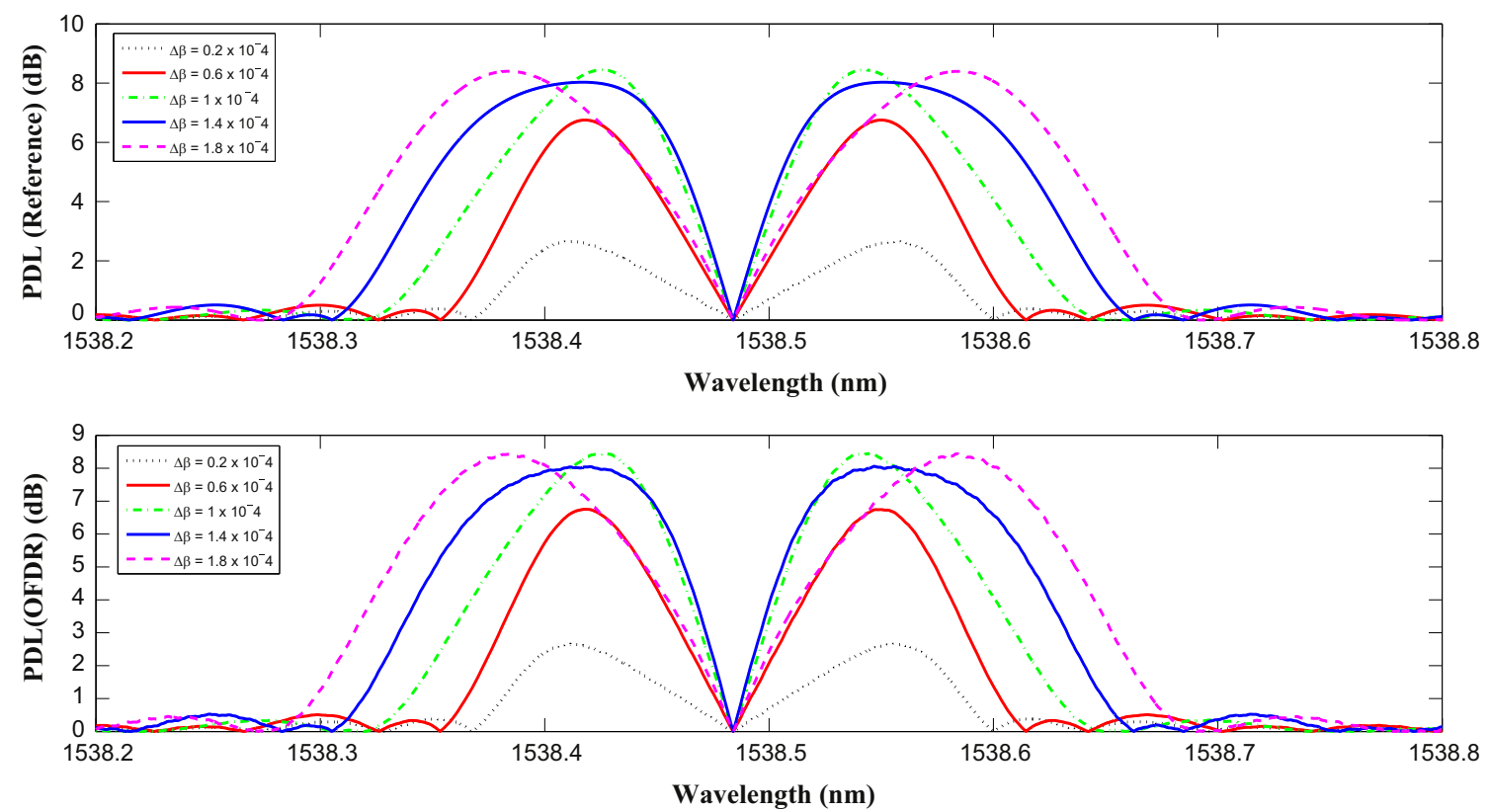

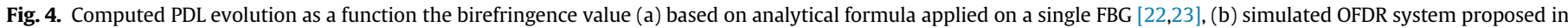
the current work.

composite structures, the simulated configuration in this paper will be experimentally implemented in the following publications.

\section{Acknowledgements}

The authors would like to express their thanks to Dr. Christophe Caucheteur and Dr. Marc Wuilpart for valuable discussions.

\section{References}

[1] Melle S, Liu K, Measures R. A passive wavelength demodulation system for guided wave Bragg grating strain sensor. IEEE Photonics Technol Lett 1992;4:516-8.

[2] Kersey AD, Berkoff T, Morey W. Fiber optic Bragg grating sensor with driftcompensated high resolution interferometric wavelength shift detection. Opt Lett 1993;5:72.

[3] Todd MD, Johnson GA, Althouse BL. A novel Bragg grating sensor interrogation system utilizing a scanning filter, a Mach-Zehnder interferometer and a $3 \times 3$ coupler. Meas Sci Technol 2001;12:771-7.

[4] Seim JM, Schulz WL, Udd E, Corona-Bittick K, Dorr J, Slattery KT. Low-cost high-speed fiber optic grating demodulation system for monitoring composite structures. In: Proceedings of SPIE, vol. 3326; 1998. p. 390.

[5] Yüksel K, Wuilpart M, Moeyaert V, Mégret P. Optical frequency-domain reflectometry: a review. In: Proceedings of the international conference on transparent optical networks (ICTON 2009), 28 June-02 July, Ponta-DelgadaAzores (P); 2009. Invited, Tu.C2.5.

[6] Kinet D, Yüksel K, Caucheteur C, Garray D, Wuilpart M, Narbonneau F, et al. Structural health monitoring of composite materials with fibre Bragg gratings interrogated by optical frequency domain reflectometer. In: Proceedings of the European conference on composite materials (ECCM 2012), 24-28 June, Venice (I); 2012.

[7] Yüksel K, Mégret P, Wuilpart M. A quasi-distributed temperature sensor interrogated by optical frequency-domain reflectometer. Meas Sci Technol 2011:22:115204.

[8] Brodzeli Z, Baxter GW, Collins SF, Canning J, Stevenson M, Buryak A. Low cost interrogation technique for a FBG sensor for combined transverse and longitudinal strain measurement. In: Proceedings of SPIE, vol. 7004; 2008. p. 700466-700466-4.

[9] Sulejmani S, Sonnenfeld C, Geernaert T, Berghmans F, Thienpont H, Eve S, et al. Towards micro-structured optical fiber sensors for transverse strain sensing in smart composite materials. IEEE Sens; 2011. p. 109-12. 10.1109/ICSENS.2011.6127305.
[10] Caucheteur C, Bette S, Garcia-Olcina R, Wuilpart M, Sales S, Capmany J, et al. Transverse strain measurements using the birefringence effect in fiber Bragg gratings. IEEE Photonics Technol Lett 2007;19:966-8.

[11] Kinet D, Mégret P, Goossen KW, Heider D, Caucheteur C. Fiber Bragg grating sensors toward structural health monitoring in composite materials: challenges and solutions. Sensors 2014;14:7394-419.

[12] Wada D, Murayama H, Igawa H. Structural health monitoring by using fiberoptic distributed strain sensors with high spatial resolution. Photonic Sens 2013;3:355-76.

[13] Wada D, Murayama H. Analytical investigation of response of birefringent fiber Bragg grating sensors in distributed monitoring system based on optical frequency domain reflectometry. Opt Lasers Eng 2014;52:99-105.

[14] Wada D, Murayama H, Igawa H. Lateral load measurements based on a distributed sensing system of optical frequency-domain reflectometry using long-length fiber Bragg gratings. J Lightw Technol 2012;52:2334-7.

[15] Wada D, Murayama H, Igawa H. Distributed monitoring of fiber Bragg gratings under local lateral loads using optical frequency-domain reflectometry. In: Proceedings of SPIE, vol. 8451; 2012. p. 84213X-1-4.

[16] Wang Y, Wang M, Huang X. Distributed load sensor using a fiber Bragg grating and its differential group delay analysis. Opt Quantum Electron 2012;44:483-91.

[17] Andreas Othonos, Kyriacos Kalli. Fiber Bragg Gratings: Fundamentals and Applications in Telecommunications and Sensing, 1998. P. 433, ISBN: 978-089006-344-6.

[18] Froggat M, Moore J. High-spatial-resolution distributed strain measurement in optical fiber with Rayleigh scatter. Appl Opt 1998;10:1735-40.

[19] Abdi AM, Suzuki S, Schülzgen A, Kost AR. Modelling, design, fabrication, and testing of a fiber Bragg grating strain sensor array. Appl Opt 2007;46:2563-74.

[20] Yüksel K, Wuilpart M, Mégret P. Analysis and suppression of nonlinear frequency modulation in an optical frequency-domain reflectometer. Opt Express 2009; 17(7):5845-51.

[21] Yüksel K, Wuilpart M, Moeyaert V, Mégret P. Complete analysis of multireflection and spectral-shadowing crosstalks in a quasi-distributed fibre sensor interrogated by OFDR. IEEE Sens J 2011;17.

[22] Caucheteur C. Realisation of mechanical and chemical sensors based on the fiber Bragg gratings technology. UMONS library [PhD thesis], chapter 5 (Study of the polarization properties of uniform fiber Bragg gratings); 2007.

[23] Caucheteur C, Bette S, Garcia-Olcina R, Wuilpart M, Sales S, Mégret P. Influence of the grating parameters on the polarization properties of fiber Bragg gratings. J Lightw Technol 2009;27:8.

[24] Lammens N, Kinet D, Chah K, Luyckx G, Caucheteur C, Degrieck J, et al, Residual strain monitoring of out-of-autoclave cured parts by use of polarization dependent loss measurements in embedded optical fiber Bragg gratings. Compos Part A: Appl Sci Manuf 2013;52:38-44. 